\title{
Autism in Adulthood: The New Home for Our Emerging Field
}

\author{
Christina Nicolaidis, $\mathrm{MD}, \mathrm{MPH}^{1-3}$
}

\begin{abstract}
A Bout a DECADE AGO, when I mentioned to a colleague that I had received an autism-related research grant, a perplexed look came across his face. "But you aren't a pediatrician, are you?" he asked. Although I wanted to respond with a snarky remark about autistic children ${ }^{*}$ not just vanishing into thin air when they turn 21 , I knew his confusion was perfectly reasonable. The overwhelming majority of autism research, services, and advocacy has focused on children. As an internal medicine physician, I care for primarily middle-aged and older adults. Autism - and autistic adults-has existed throughout history, likely with little change in actual prevalence over time, ${ }^{1}$ but the Diagnostic and Statistical Manual of Mental Disorders (DSM) only first mentioned autism in 1980 and Asperger's disorder in 1995, long after most of my patients had grown up. Before my own child was diagnosed with autism in 2004, I only knew of two autistic adults on my entire panel of patients.

In the intervening years, a lot has changed. As the large cohort of children who were diagnosed with autism in the late 1990s and early 2000s has started to transition into adulthood, the need for research, services, and policies focused on adults is no longer in question. In the United States, for example, the Interagency Autism Coordinating Committee has clearly stressed the importance of meeting the needs of autistic adults ${ }^{2}$ and the National Institutes of Health (NIH) have issued multiple requests for proposals on autism in adulthood. When Dora Raymaker and I cofounded the Academic Autism Spectrum Research Partnership in Research and Education (www.aaspire.org) in $2006,{ }^{3}$ the idea of including autistic adults as equal partners on the research team seemed preposterous to many reviewers. Now, similar partnerships are forming in the United Kingdom
\end{abstract}

*We understand that there are strong arguments and passions on both sides of the debate between the use of person-first language (e.g., "person with autism") and identity-first language (e.g., "autistic person"). However, there is a growing body of scientific and community literature documenting the dislike, among autistic individuals, of person-first language and its potential for increasing stigma. ${ }^{7,8}$ Based on this literature and the strong preference of the autistic members of our Editorial Board, our journal's policy strongly recommends either using identity-first language or more neutral terms such as "person on the autism spectrum." (www.shapingautismresearch.co.uk) and Australia (www .autismcrc.com.au) and a growing number of funders, organizations, and researchers actively recognize the need to listen to and partner with autistic adults in research and policy. Research on autism in adulthood is increasingly appearing in the literature of multiple disciplines. And almost every week, I notice a story in the popular media related to autism and employment or other issues that affect adults. As a clinician, researcher, parent, and friend, I know that we still have a very long way to go, but I am optimistic that the field is moving rapidly in the right direction.

Just as emerging adults may reach a point wherein they are ready to establish a home of their own, this is the time for us, as a field, to establish our own home-a home for research and scholarship on the most pressing issues affecting autistic adults, from emerging adulthood to later life. Together, we will strive to make our new home inclusive, respectful, and vibrant, with high standards for rigorous research and ample space for new perspectives, in-depth analysis, and interprofessional dialogue. We will build a new type of journal that truly integrates the priorities and contributions of autistic adults into the peer-reviewed literature. The goal is to create a strong evidence base that promotes practice, systems, policy, and societal change. I envision Autism in Adulthood as the academic home for the research and scholarship that will ultimately enable all autistic adults to lead healthy, fulfilling lives.

For decades, autistic adults and their allies have been calling for such research. One high-priority area is the mental and physical health of autistic adults. Autistic individuals have decreased life expectancy, ${ }^{4}$ with high rates of co-occurring physical and mental health conditions, ${ }^{5}$ and known disparities in healthcare. ${ }^{6}$ In this Inaugural Issue, Zerbo et al. present results from a large integrated health system, noting that autistic adults had increased rates of healthcare utilization and costs across multiple domains, but that autistic women had decreased rates of gynecologic health visits or cervical screening. Two Perspectives in this Inaugural Issue explore where we should go from here: Warner et al. describe the outcome of a 2-day summit in the United Kingdom where researchers, autistic adults, and other stakeholders identified priorities for future research on the physical health of autistic

\footnotetext{
${ }^{1}$ Editor-in-Chief, Autism in Adulthood.

${ }^{2}$ Regional Research Institute, School of Social Work, Portland State University, Portland, Oregon.

${ }^{3}$ Department of Medicine, Oregon Health \& Science University, Portland, Oregon.
} 
adults and Maddox and Gaus offer their vision for improved community mental health services for autistic adults in the United States.

The Inaugural Issue also offers a taste of some of the other high-priority areas that we expect to cover in Autism in Adulthood. Conner et al.'s study highlights the limitations of existing measures for diagnosing autism in adults. You will also find four insightful qualitative studies, one by Kirby et al. about the perspectives of autistic youth on adulthood, one by Sosnowy et al. on how young autistic adults seek friendship, one by Kim and Bottema-Beutel on the negotiation of identity in the online discourse of autistic adults; and one by Kourti and McLeod on views about gender identity among an online group of autistic adults raised as girls. We actively encourage submissions from autistic authors and other stakeholders. In addition to scientific articles authored by autistic researchers (e.g., the study that was first authored by Dr. Kourti), we plan to include at least one personal essay from an autistic author in each issue. In this issue, Becca Lory vividly describes her experiences as a participant in autism research and challenges researchers to think about how their study procedures and payments affect autistic adults. We also plan to regularly include round table discussions on issues that are critical to autistic adults. In this issue, four experts, including two autistic scholars, discuss controversies about the relationship between autism and empathy.

We expect future issues to cover a wide range of topics of relevance to autistic adults and their families. As a multidisciplinary, interprofessional journal, we welcome research and scholarship across the translational spectrum, from basic science research with implications for autism in adulthood to studies of aging; to research that helps us better understand autistic intelligence, sensory processing, communication, executive functioning, and flexibility; to studies on social services, employment, housing, education, community participation, economic well-being, and quality of life; and to policies and programs affecting adults on the autism spectrum, human and disability rights, ethical issues, and the portrayal of adult autism in society and the media. We strongly welcome work on the intersectionality of autism and race, ethnicity, nationality, gender, sexual orientation, disability, class, trauma, discrimination, and other social determinants of health, as well as articles addressing the wide heterogeneity of the autism spectrum, including studies focused on minimally verbal autistic adults and those with high-support needs.

The success of any endeavor, journals included, depends on people. My long-time friend and collaborator, Dr. Raymaker, serves as the Associate Editor of Autism in Adulthood. She brings a dual perspective, both as an autistic adult and as a new NIH-funded autism researcher. I am proud to have assembled a highly engaged, accomplished, multidisciplinary, international Editorial Board, all of whom bring expertise as researchers, scholars, clinicians, advocates, and community leaders. Ten Editorial Board members openly identify as being on the autism spectrum and many bring personal experience as family members, caregivers, friends, and allies of autistic individuals. As members of an emerging field, we all come from different "families of origin," with different training, experiences, cultural norms, rules, lenses, and approaches. As we have tried to bring together the health and social sciences (let alone as we have tried to include contributions from the humanities or outside of academia), we have quickly realized that even our basic assumptions about the peer-review process or journal style often differ. Yet, these differences offer an exciting opportunity to set new norms for our emerging field. Already, our Editorial Board has surpassed all my expectations, providing thoughtful, expert guidance, and working hard to assemble this issue. We are fortunate to be working with Mary Ann Liebert, Inc., experienced, mission-driven publishers who recognized the pressing need for a journal on autism in adulthood and have actively supported our innovative approach and dedication to inclusion.

And of course, it is reviewers who make a journal "peerreviewed." Given our commitments to scientific rigor, inclusion, relevance, and respect, we are using an innovative process that combines a traditional scientific peer review with an "autistic review." Each article will be reviewed by two or more academic experts and at least one autistic adult. Although on occasion, an autistic researcher may fill both roles, we expect the majority of autistic reviews to be by lay people. These autistic reviewers will help ensure that work published in our journal is relevant to the needs of the autistic community, uses respectful concepts and language, and includes lay summaries that are accessible to autistic adults, their families, and other stakeholders. We have found that the autistic community is more than ready, able, and willing to play a greater role in the peer-reviewed literature. Within days of advertising our desire to include autistic adults in this review process, we had approximately 200 autistic adult volunteers. Already, reviews by autistic adults have strengthened the articles in our Inaugural Issue. We look forward to refining and strengthening our autistic review process and to finding ways to make our journal even more inclusive, respectful, and relevant in the future.

As this Inaugural Issue goes to print, I am filled with gratitude and excitement. Back in 2006, when I first started collaborating with autistic adults, I was struck by the lack of inclusion of autistic perspectives in the autism literature and the dearth of research on issues affecting adults. As my own child now approaches adulthood, I cannot help but notice how much the field has evolved-and how much more we have to do. Please join me in making Autism in Adulthood the new home for our emerging field. Together, we can play a critical role in shaping the literature that drives positive change for all autistic adults.

\section{References}

1. Hill AP, Zuckerman K, Fombonne E. Epidemiology of autism spectrum disorders. In: Maria de los Angeles Robinson-Agramonte, ed. Translational Approaches to Autism Spectrum Disorder. Switzerland: Springer; 2015; 13-38.

2. Interagency Autism Coordinating Committee. 2016-2017 Interagency Autism Coordinating Committee Strategic Plan for Autism Spectrum Disorder. 2017. U.S. Department of Health and Human Services Interagency Autism Coordinating Committee. https://iacc.hhs.gov/publications/strategic-plan/ 2017/

3. Nicolaidis C, Raymaker D, McDonald K, et al. Collaboration strategies in nontraditional community-based participatory research partnerships: lessons from an academic-community 
partnership with autistic self-advocates. Prog Community Health Partnersh. 2011;5(2):143-150.

4. Hirvikoski T, Mittendorfer-Rutz E, Boman M, Larsson $\mathrm{H}$, Lichtenstein P, Bölte S. Premature mortality in autism spectrum disorder. Br J Psychiatry. 2016;208(3): 232-238.

5. Croen LA, Zerbo O, Qian Y, et al. The health status of adults on the autism spectrum. Autism. 2015;19(7):814-823.

6. Nicolaidis C, Raymaker D, McDonald K, et al. Comparison of healthcare experiences in autistic and non-autistic adults:
A cross-sectional online survey facilitated by an academiccommunity partnership. J Gen Intern Med. 2013;28(6): 761-769.

7. Gernsbacher MA. Editorial perspective: The use of personfirst language in scholarly writing may accentuate stigma. $J$ Child Psychol Psychiatry. 2017;58(7):859-861.

8. Kenny L, Hattersley C, Molins B, Buckley C, Povey C, Pellicano E. Which terms should be used to describe autism? Perspectives from the UK autism community. Autism. 2016; 20(4):442-462. 